\title{
Relation between peak value and acoustic damping of sound pressure level of one dimensional sound field partitioned with perforated plates
}

\author{
Kunihiko ISHIHARA, Akari GOTO, Makoto KASHINO
}

\begin{abstract}
In an industry field, the acoustic characteristics such as a resonance frequency and an acoustic damping ratio are often required. The response test due to a random noise wave and the sweep test due to a sinusoidal wave can be performed in those cases. In vibration theory, it is well known that the peak value at resonance is inversely proportional to the damping ratio. Is it true even in the acoustic theory? If it can be true the acoustic damping ratio can be obtained simply by the peak sound pressure level after the relation between the peak value and the damping ratio is examined only once. In this study, the sound pressure level of the one dimensional duct with the perforated plate will be calculated by the transfer matrix method in various parameters such as the aperture ratio, the hole's length and the set position of the plate. These calculations are conducted for the first mode, the second mode and third mode. And the relation between the peak value and the damping ratio are examined.
\end{abstract}

Index Terms - Acoustic characteristics, Damping ratio, Resonance frequency, Transfer matrix method, Perforated plate

\section{INTRODUCTION}

A perforated plate is widely used as an acoustic absorption material for compressors [1] and acoustic barriers for roads and railways[2]. It has been confirmed that the perforated plate suppressed the self- sustained tone generated from heat exchangers like a boiler by using it on a duct wall[3] [6]. The effects of the perforated plate on the acoustic natural frequency of an one dimensional sound field partitioned with the perforated plate has been studied in our previous investigations [7],[8]. It was clarified that the acoustic natural frequency of the one dimensional sound field partitioned with the perforated plate becomes lower as the aperture ratio becomes smaller. The acoustic characteristics such as a resonant frequency and an acoustic damping ratio of an one dimensional sound field like a duct has often been required. In that case, the random excitation or the sine wave sweep test has been performed to obtain the acoustic characteristics.

By the way, we have a question that whether the response value at resonance is inversely proportional to the acoustic

damping ratio similar to the vibration theory or not. On the other hand, we have the thought it is natural. But it is not clear in the present stage. If it is possible, the peak value of the sound pressure level at resonance can be obtained easily without obtaining the acoustic damping ratio which is hard to obtain.

Kunihiko Ishihara, Department of Health and Welfare, Tokushima Bunri University, Shido, Sanuki-city, Kagawa, Japan, +81878997247

Akari Goto, Department of Health and Welfare, Tokushima Bunri University, Shido, Sanuki-city, Kagawa, Japan, +81878997257

Makoto Kashino, Department of Health and Welfare, Tokushima Bunri University, Shido, Sanuki-city, Kagawa, Japan, +81878997258
Then, in this study, the sound pressure level is calculated for each parameter of the perforated plate such as the aperture ratio, hole's length and the setting position in the duct and the peak value and acoustic damping ratio for $1^{\text {st }}$, $2^{\text {nd }}$ and $3^{\text {rd }}$ modes are obtained by the analysis. The relation between the peak value and the acoustic damping will be examined. The analysis is performed by using the Transfer Matrix Method and Melling's results[9],[10].

\section{ANALYTICAL METHOUD}

Figure 1 shows an analytical model of the duct with a perforated plate. The numbers show the varying points of the cross section of the duct and the perforated plate position. Indicating the state vector at each numbering position as $\left[\begin{array}{ll}P_{i} & U_{i}\end{array}\right]^{T} \quad(i=1 \sim 4)$, the equation (1) can be obtained as the relation between both state vectors of first and terminal positions. Where $P_{i}, U_{i}$ are the sound pressure and the volume velocity respectively.

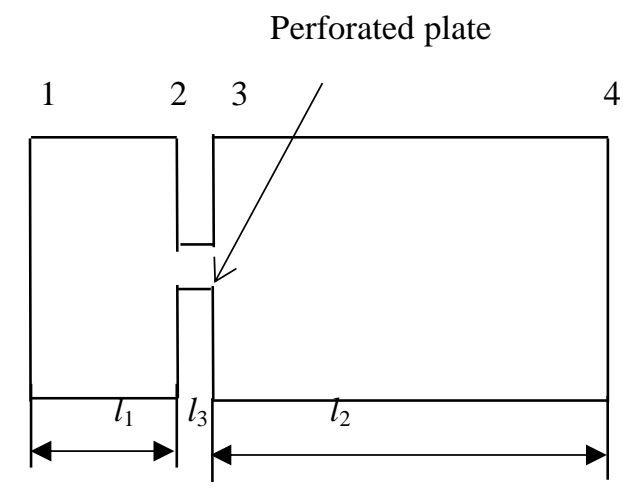

Fig.1 Analytical model of 1D duct

$\left[\begin{array}{l}P_{4} \\ \mathrm{U}_{4}\end{array}\right]=\left[\begin{array}{cc}\cos k l_{2} & j Z_{2} \sin k l_{2} \\ j \frac{1}{Z_{2}} \sin k l_{2} & \cos k l_{2}\end{array}\right]\left[\begin{array}{cc}\cos k l_{3} & j Z_{3} \sin k l_{3} \\ j \frac{1}{Z_{3}} \sin k l_{3} & \cos k l_{3}\end{array}\right]$
$\times\left[\begin{array}{cc}\cos \mathrm{kl}_{1} & \mathrm{jZ}_{1} \sin \mathrm{kl}_{1} \\ \mathrm{j} \frac{1}{\mathrm{Z}_{1}} \sin \mathrm{kl}_{1} & \cos \mathrm{kl}_{1}\end{array}\right]\left[\begin{array}{l}\mathrm{P}_{1} \\ \mathrm{U}_{1}\end{array}\right]=\left[\begin{array}{ll}\mathrm{A} 11 & \mathrm{~A} 12 \\ \mathrm{~A} 21 & \mathrm{~A} 22\end{array}\right]\left[\begin{array}{l}\mathrm{P}_{1} \\ \mathrm{U}_{1}\end{array}\right]$

Given the unit forced displacement at the left end of the duct and the boundary condition of the right end is given as follows because the right end of the duct is closed.

$$
U_{1}=1, \quad U_{4}=0
$$

And the center matrix of the equation (1) which relates the state vectors of before and behind the perforated plate can be given as follows. 
Relation between peak value and acoustic damping of sound pressure level of one dimensional sound field partitioned with perforated plates

$$
\left[\begin{array}{cc}
1 & Z_{R}+j Z_{i} \\
0 & 1
\end{array}\right] \quad \text { Where } \mathrm{Z}=\mathrm{z} / \mathrm{S}
$$

Here Melling's equation (4) is used as the acoustic impedance before and behind the perforated plate.

$$
\begin{aligned}
z & =\left(2 \mu l_{3} / r_{0}\right) \sqrt{\omega \rho / 2 \mu}(1+j)+j \omega \rho l_{3} \\
& =\left(2 \mu l_{3} / r_{0}\right) \sqrt{\omega \rho / 2 \mu}+j \omega \rho l_{3}\left[1+\frac{1}{r_{0}} \sqrt{\frac{2 \mu}{\omega \rho}}\right]
\end{aligned}
$$

As a result, $\mathrm{A} 11 \sim \mathrm{A} 22$ of equation (1) become as follows.

$$
\begin{gathered}
\mathrm{A} 11=\cos k l_{2} \cos k l_{1}-\frac{Z_{i}}{Z_{1}} \\
\cos k l_{2} \sin k l_{1}-\frac{Z_{2}}{Z_{1}} \sin k l_{2} \sin k l_{1} \\
+j \frac{Z_{R}}{Z_{1}} \cos k l_{2} \sin k l_{1}
\end{gathered}
$$

$$
\begin{aligned}
& \mathrm{A} 12=Z_{R} \cos k l_{2} \cos k l_{1} \\
&+j\left[Z_{1} \cos k l_{2} \sin k l_{1}+Z_{i} \cos k l_{2} \cos k l_{1}+Z_{2} \sin k l_{2} \cos k l_{1}\right] \\
& \mathrm{A} 21=-\frac{Z_{R}}{Z_{1} Z_{2}} \sin k l_{2} \sin k l_{1}+j\left[\frac{1}{Z_{2}} \sin k l_{2} \cos k l_{1}\right. \\
&\left.-\frac{Z_{i}}{Z_{2} Z_{1}} \sin k l_{2} \sin k l_{1}+\frac{1}{Z_{1}} \sin k l_{1} \cos k l_{2}\right]
\end{aligned}
$$

$$
\begin{aligned}
\mathrm{A} 22= & {\left[\cos k l_{2} \cos k l_{1}-\frac{Z_{1}}{Z_{2}} \sin k l_{2} \sin k l_{1}-\frac{Z_{i}}{Z_{2}} \sin k l_{2} \cos k l_{1}\right] } \\
& +j \frac{Z_{R}}{Z_{2}} \sin k l_{2} \cos k l_{1}
\end{aligned}
$$

The equation (5) can be obtained as the sound pressure P4 of the right end of the duct by using these equations.

$$
\mathrm{P} 4=\frac{-\mathrm{A}_{11} \mathrm{~A}_{22}+\mathrm{A}_{12} \mathrm{~A}_{21}}{\mathrm{~A}_{21}}
$$

The sound pressures were calculated for the aperture ratios $1 \%, 4 \%$ and $16 \%$. And calculated for the hole' length $l_{3}$ of $0.0023 \mathrm{~m}, 0.0046 \mathrm{~m}, 0.1 \mathrm{~m}$ and $0.2 \mathrm{~m}$ for each aperture ratio. Moreover we calculated the sound pressures for three perforated plate positions (1) $l_{1}=0.334 \mathrm{~m}, l_{2}=0.5 \mathrm{~m}, \quad$ (2) $l_{1}=0.434 \mathrm{~m}, \quad l_{2}=0.4 \mathrm{~m}$, (3) $l_{1}=0.634 \mathrm{~m}, \quad l_{2}=0.2 \mathrm{~m}$ for three aperture ratios and four the perforated plate positions. $r_{\mathrm{o}}$ is used the radius that makes a circle of the same area as the entire holes area. Namely $r_{0}=\sqrt{(0.2 \mathrm{~m} \times 0.2 \mathrm{~m} \times \varphi) / \pi}$. This treatment should be noticed not to be able to obtain the correct acoustic damping ratio and this treatment is only used to clarify the relationship between the peak sound pressure and the acoustic damping ratio. The frequency resolution is $10 \mathrm{~Hz}$.

\section{ANALYTICAL RESULTS}

\section{i FREQUENCY RESPONSES}

Figure $2 \sim$ Figure 4 show the frequency responses of P4 (Right end pressure) for three perforated plate positions (1), (2) and (3) described above. The left figures show the frequency responses for the aperture ratio $\varphi=1 \%$ and right figures for $\varphi=16 \%$. Notification $334 * 500$ shows the perforated plate position and this indicates the perforated plate is set at the position of $334 \mathrm{~mm}$ from the left end of the duct. Sum of two numbers is $834 \mathrm{~mm}$ constant and it is the total length of the duct.

\section{ii ACOUSTIC DAMPING RATIO}

It is well known in the vibration theory that the peak value at resonance is inversely proportional to the damping ratio. Is it true in the acoustic field like the one dimensional duct examined here? We think that it is difficult to answer the question immediately. Then the acoustic damping ratio is obtained by using the half power method. The equation of half power method is given as follows.

$$
\zeta=\Delta f / 2 / f_{\mathrm{n}}
$$

Where $\Delta f$ is frequency difference of $f_{1}$ and $f_{2}(\Delta f=$ $f_{2}-f_{1}$ ) and these are the frequencies at which the $3 \mathrm{~dB}$ lower than the peak value. $f_{\mathrm{n}}$ is the natural acoustic frequency. Suffix $n$ indicates mode order .
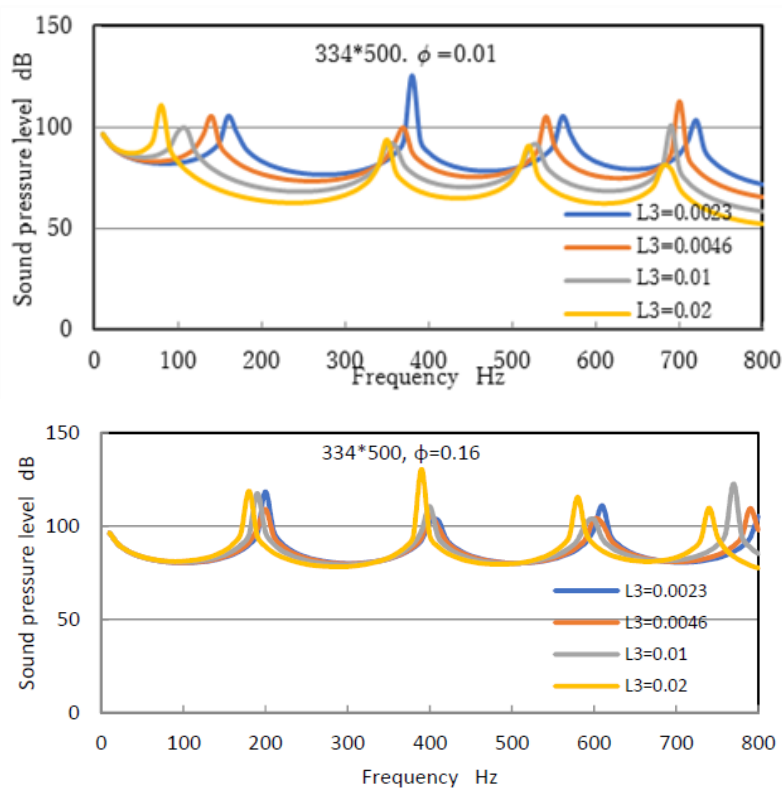

Fig.2 Frequency responses for duct of $343 * 500$
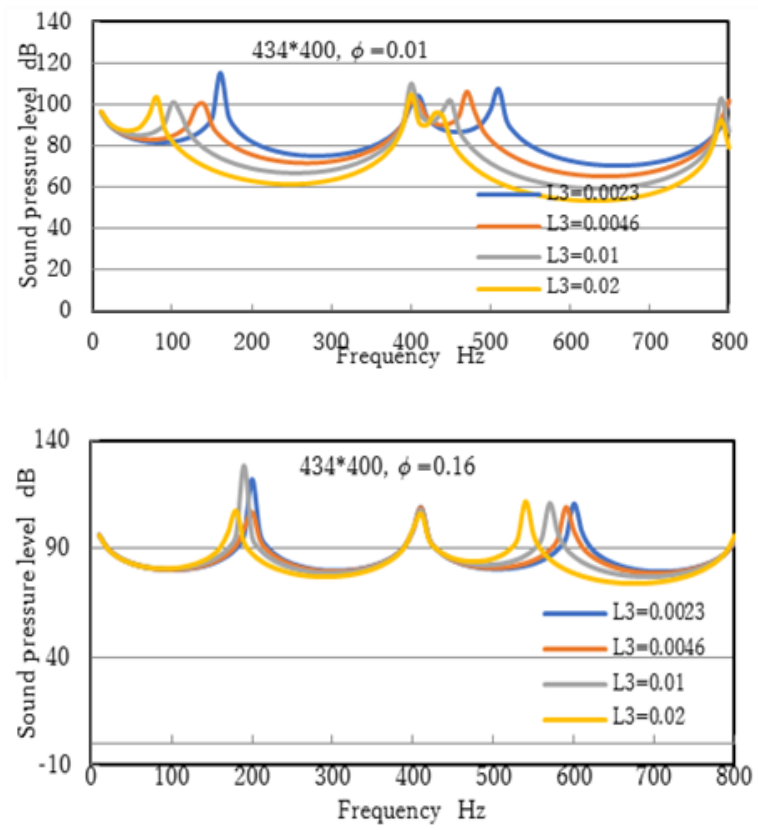

Fig. 3 Frequency responses for duct of $443 * 400$ 

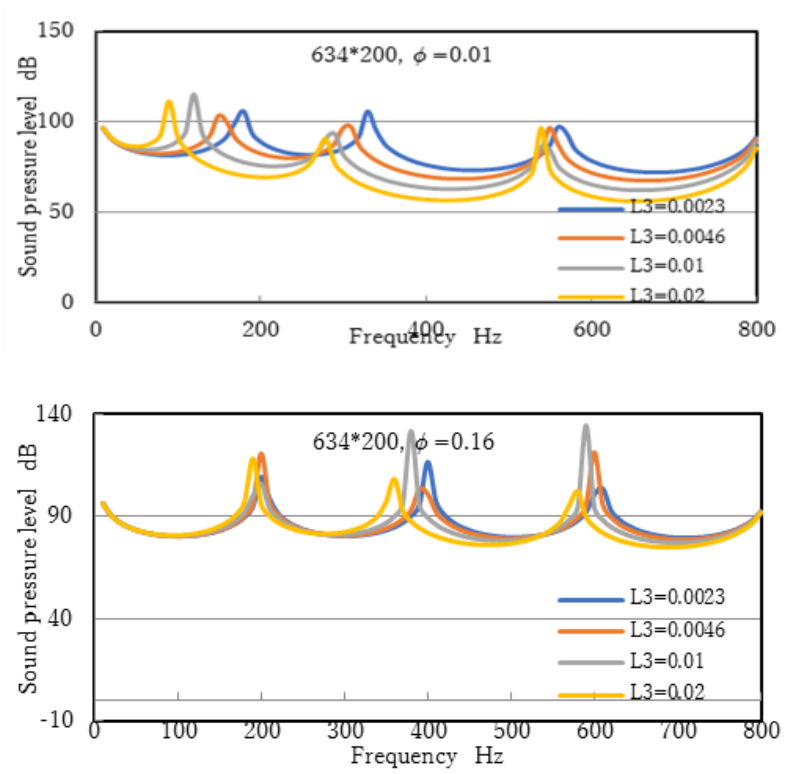

Fig.4 Frequency responses for duct of $643 * 200$

Then the calculations were performed by varying $\Delta f$ as best suited to the acoustic damping to be able to catch the each mode peak value. The calculation results of acoustic damping are shown in table1 to table6. These are the results in the case of the perforated plate position of $434 * 400$. Table 1 and 2 show the results from the peaks of $1^{\text {st }}$ mode, Table 3 and 4 from the peaks of $2^{\text {nd }}$ mode and Table 5 and 6 from the peaks of $3^{\text {rd }}$ mode. The odd number of the table show the case of $\phi=0.01$ and the even number of the table show the case of $\phi=0.16$. Where $\phi$ is the aperture ratio. The values of right column ( hatching ) show the product of the sound pressure $p$ and the acoustic damping $\zeta$. From these tables, the product value is about 3277 for $1^{\text {st }}$ mode regardless of the perforated plate positions and hole's length. And 1570 for $2^{\text {nd }}$ mode, 1094 for $3^{\text {rd }}$ mode respectively. These values are mean values of four values for L3. It can be said that the peak sound pressure $p$ is inversely proportional to the acoustic damping $\zeta$ and the product value of $p$ and $\zeta$ for each mode is inversely proportional to the mode order.

Table $1434 * 400, \phi=0.01,1^{\text {st }}$ mode

\begin{tabular}{|r|r|r|r|}
\hline \multicolumn{1}{|c|}{ L3 } & \multicolumn{1}{c|}{$\mathrm{P}(\mathrm{Pa})$} & \multicolumn{1}{c|}{$\zeta$} & $\mathrm{P} \times \zeta$ \\
\hline 0.0023 & 7103951.7 & 0.000446 & 3165 \\
\hline 0.0046 & 3767038 & 0.000892 & 3360 \\
\hline 0.01 & 2016044 & 0.00158 & 3185 \\
\hline 0.02 & 1339676.7 & 0.002493 & 3340 \\
\hline
\end{tabular}

Table $2434 * 400, \phi=0.16,1^{\text {st }}$ mode

\begin{tabular}{|r|c|c|r|}
\hline \multicolumn{1}{|c|}{ L3 } & $\mathrm{P}(\mathrm{Pa})$ & $\zeta$ & $\mathrm{P} \times \zeta$ \\
\hline 0.0023 & 54890856 & $6.48 \mathrm{E}-05$ & 3559 \\
\hline 0.0046 & 27669417 & 0.000114 & 3158 \\
\hline 0.01 & 13091819 & 0.000237 & 3104 \\
\hline 0.02 & 6990367.4 & 0.000478 & 3344 \\
\hline
\end{tabular}

Table $3434 * 400, \phi=0.01,2^{\text {nd } t}$ mode

\begin{tabular}{|r|c|c|r|}
\hline \multicolumn{1}{|c|}{$\mathrm{L} 3$} & $\mathrm{p}(\mathrm{Pa})$ & $\zeta$ & $\mathrm{P} \times \zeta$ \\
\hline 0.0023 & 295120923 & $5.40 \mathrm{E}-06$ & 1594 \\
\hline 0.0046 & 147570653 & $1.11 \mathrm{E}-05$ & 1632 \\
\hline 0.01 & 69502432 & $2.22 \mathrm{E}-05$ & 1541 \\
\hline 0.02 & 36475395 & $3.96 \mathrm{E}-05$ & 1444 \\
\hline
\end{tabular}

Table $4434 * 400, \phi=0.16,2^{\text {nd }}$ mode

\begin{tabular}{|r|c|c|c|}
\hline \multicolumn{1}{|c|}{$\mathrm{L} 3$} & $\mathrm{p}(\mathrm{Pa})$ & $\zeta$ & $\mathrm{P} \times \zeta$ \\
\hline 0.0023 & $2.35 \mathrm{E}+09$ & $6.74 \mathrm{E}-07$ & 1585 \\
\hline 0.0046 & $1.178 \mathrm{E}+09$ & $1.35 \mathrm{E}-06$ & 1589 \\
\hline 0.01 & 543876132 & $2.95 \mathrm{E}-06$ & 1602 \\
\hline 0.02 & 273526873 & $5.78 \mathrm{E}-06$ & 1580 \\
\hline
\end{tabular}

Table $5434 * 400, \phi=0.01,3^{\text {rd }}$ mode

\begin{tabular}{|r|c|c|c|}
\hline \multicolumn{1}{|c|}{$\mathrm{L} 3$} & $\mathrm{p}(\mathrm{Pa})$ & $\zeta$ & $\mathrm{P} \times \zeta$ \\
\hline 0.0023 & 4560369.2 & 0.000235 & 1072 \\
\hline 0.0046 & 2834653.6 & 0.0003757 & 1065 \\
\hline 0.01 & 2333458.1 & 0.0004767 & 1112 \\
\hline 0.02 & 2760577.9 & 0.0004048 & 1117 \\
\hline
\end{tabular}

Table $6434 * 400, \phi=0.16,3^{\text {rd }}$ mode

\begin{tabular}{|r|r|r|r|}
\hline \multicolumn{1}{|c|}{$\mathrm{L} 3$} & $\mathrm{p}(\mathrm{Pa})$ & $\zeta$ & $\mathrm{P} \times \zeta$ \\
\hline 0.0023 & 32998941 & $3.29 \mathrm{E}-05$ & 1084 \\
\hline 0.0046 & 16904409 & $6.59 \mathrm{E}-05$ & 1113 \\
\hline 0.01 & 8521185.1 & 0.0001268 & 1081 \\
\hline 0.02 & 5432503.3 & 0.0002032 & 1104 \\
\hline
\end{tabular}

\section{PHYSICAL NUDERSTANDING DUE TO EQUATION}

Consider the lateral vibration of a bar which is equivalent to the acoustic phenomenon of an one dimensional sound field like the duct. The equation of motion without damping can be described by the equation (7).

$$
-\rho A \frac{\partial^{2} u}{\partial t^{2}}+A E \frac{\partial^{2} u}{\partial x^{2}}=0
$$

Where $u$ is the displacement.

Consider the viscous coefficient $\mathrm{c}\left(\mathrm{N} \cdot \mathrm{s} / \mathrm{m}^{3}\right)$ per unit area on this equation, we got the next equation.

$$
-\rho A \frac{\partial^{2} u}{\partial t^{2}}-c A \frac{\partial u}{\partial t}+A E \frac{\partial^{2} u}{\partial x^{2}}=0
$$


Relation between peak value and acoustic damping of sound pressure level of one dimensional sound field partitioned with perforated plates

Dividing both sides by $\rho A$

$$
\frac{\partial^{2} u}{\partial t^{2}}+\gamma \frac{\partial u}{\partial t}-\frac{E}{\rho} \frac{\partial^{2} u}{\partial x^{2}}=0 \quad \text { Where } \gamma=\frac{c}{\rho}
$$

Here putting $a^{2}=\frac{E}{\rho}$

$$
\frac{\partial^{2} u}{\partial t^{2}}+\gamma \frac{\partial u}{\partial t}-a^{2} \frac{\partial^{2} u}{\partial x^{2}}=0
$$

Putting $u_{i}(x, t)=\varphi_{i}(x) \cdot q_{i}(t) \quad(i:$ mode order $)$ and substituting this to the equation (9)

$$
\varphi_{i} \frac{\mathrm{d}^{2} q_{i}}{\mathrm{~d} t^{2}}+\gamma \varphi_{i} \frac{\mathrm{d} q_{i}}{\mathrm{~d} t}-a^{2} \frac{\mathrm{d}^{2} \varphi_{i}}{\mathrm{~d} x^{2}} \cdot q_{i}=0
$$

Multiplying $\varphi_{j}$ (Mode function) to both sides and integrating $0 \sim 1$, We got the next equation.

$$
\int_{0}^{l} \varphi_{i} \varphi_{j} \cdot \mathrm{d} x \frac{\mathrm{d}^{2} q_{i}}{\mathrm{~d} t^{2}}+\gamma \int_{0}^{l} \varphi_{i} \varphi_{j} \cdot \mathrm{d} x \frac{\mathrm{d} q_{i}}{\mathrm{~d} t}-a^{2} \int_{0}^{l} \frac{\mathrm{d}^{2} \varphi_{i}}{\mathrm{~d} x^{2}} \cdot \varphi_{j} \cdot \mathrm{d} x \cdot q_{i}=0
$$

From the orthogonality of the mode, $\int_{0}^{l} \varphi_{i} \varphi_{j} \cdot \mathrm{d} x= \begin{cases}i \neq j & 0 \\ i=j & A\end{cases}$

And performing the partial integration and considering the boundary conditions $\varphi_{i}(0)=\varphi_{j}(l)=0$

$$
\int_{0}^{l} \frac{\mathrm{d}^{2} \varphi_{i}}{\mathrm{~d} x^{2}} \cdot \varphi_{j} \cdot \mathrm{d} x=\left.\frac{\mathrm{d} \varphi_{i}}{\mathrm{~d} x} \cdot \varphi_{j}\right|_{0} ^{l}-\int_{0}^{l} \frac{\mathrm{d} \varphi_{i}}{\mathrm{~d} x} \cdot \frac{\mathrm{d} \varphi_{j}}{\mathrm{~d} x} \mathrm{~d} x=-\int_{0}^{l}\left(\frac{\mathrm{d} \varphi_{i}}{\mathrm{~d} x}\right)^{2} \mathrm{~d} x=-\mathrm{B}
$$

Then

$$
\begin{aligned}
& A_{i} \frac{\mathrm{d}^{2} q_{i}}{\mathrm{~d} t^{2}}+\gamma A_{i} \frac{\mathrm{d} q_{i}}{\mathrm{~d} t}+a^{2} B_{i} \cdot q_{i}=0 \\
& \frac{\mathrm{d}^{2} q_{i}}{\mathrm{~d} t^{2}}+\gamma \frac{\mathrm{d} q_{i}}{\mathrm{~d} t}+a^{2} \frac{B_{i}}{A_{i}} \cdot q_{i}=0
\end{aligned}
$$

Where putting $\quad a^{2} \frac{B_{i}}{A_{i}}=\omega_{i}^{2}, \gamma=2 \zeta_{i} \omega_{i}$

$$
\frac{\mathrm{d}^{2} q_{i}}{\mathrm{~d} t^{2}}+2 \zeta_{i} \omega_{i} \frac{\mathrm{d} q_{i}}{\mathrm{~d} t}+\omega_{i}^{2} \cdot q_{i}=0
$$

We can obtain the equation of motion for one degree of freedom vibration system. The solution of the forced vibration in the case of given the steady external force to the right hand side becomes as follows.

$$
q_{i}=\frac{f_{i} / m_{i} \omega_{i}{ }^{2}}{\sqrt{\left(1-u_{i}{ }^{2}\right)^{2}+\left(2 \zeta_{i} u_{i}\right)^{2}}}
$$

Where $f_{i}=\int_{0}^{l} F \cdot \varphi_{i} \mathrm{~d} x$

The steady state solution can be obtained as follows.

$$
u_{i}(x, t)=\varphi_{i}(x) \cdot q_{i}(t)
$$

From the above theoretical development, the damping ratio $\zeta_{i}$ of mode $i$ is inversely proportional to the natural circular frequency $\omega_{i}$ due to $\gamma=2 \zeta_{i} \omega_{i}$ when $\gamma$ is constant. And the damping ratio becomes inversely proportional to the mode order as the natural frequency is proportional to the mode order. This is coincident with the result derived from the calculation result. As a result, it was reasonable that the damping to the one dimensional sound field partitioned with the perforated plate can be given like the equation (8).

The mode orthogonality as shown in the equation (11) is even true for the case of one dimensional sound field partitioned with the perforated plate. Figure5 (a) and (b) are $1^{\text {st }}$ mode and $2^{\text {nd }}$ mode shapes of the pressure obtained by the reference [6] respectively. The horizontal axis shows the position from the origin and the vertical axis shows the normalized sound pressure. The length of this duct is $500 \mathrm{~mm}$. It can be seen from this figure that the sound pressure suddenly drops at the position of the perforated plate.

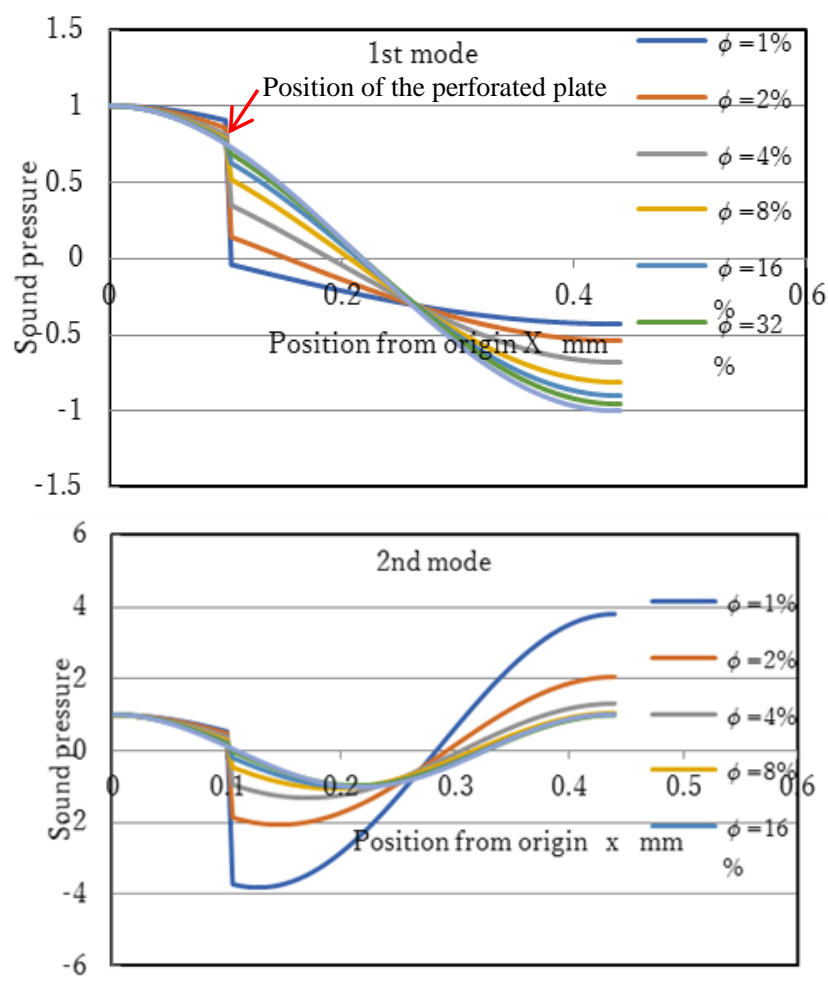

Fig. $51^{\text {st }}$ and $2^{\text {nd }}$ modes of sound pressure

The result calculated by the equation (5) shows that the value in the case of $i=j$ is $1 / 100$ order smaller than that in the case of $\mathrm{i}=\mathrm{j}$. Namely the orthogonal condition is true for this case.

Table7 Verification of orthogonality condition

\begin{tabular}{|c|c|c|c|c|c|c|c|}
\hline$\varphi$ & 0.01 & 0.02 & 0.04 & 0.08 & 0.16 & 0.32 & 1 \\
\hline$i \neq j$ & -0.00212 & -0.00161 & -0.00124 & -0.00095 & -0.00073 & -0.00616 & -0.00301 \\
\hline$i=j$ & 0.127607 & 0.133895 & 0.151384 & 0.174766 & 0.194437 & 0.207706 & 0.222594 \\
\hline
\end{tabular}

\section{CONCLUSIONS}

In this paper, theoretical analysis was conducted to clarify the relation between the peak sound pressure level at resonance and the acoustic damping ratio. In general, the peak value at resonance is inversely proportional to the damping ratio in the vibration theory. This is the purpose to confirm that the peak value at resonance is inversely proportional to the damping ratio in also acoustic fields. We got the following findings from the theoretical consideration,

(1) The pressure at resonance is inversely proportional to the acoustic damping ratio in even acoustic fields. 
(2) It is true that the mode order bear an inverse relation to the acoustic damping ratio.

\section{REFERENCES}

[1] Kimura, Y., Yamaguchi, Z. and Sakatani, T., Perforated panel type silencer for screw compressors, Kobe Steel Engineering Reports, Vol.59, No.3 (2009), pp.13-16(in Japanese).

[2] Tsugihashi, K., Yamagiwa, I. and Kikuchi, M., Research on Numerical Analysis Technique for Sound Fields including Perforated Plate, Kobe Steel Engineering Report, Vol.64, No.2(2014), pp.90-95(in Japanese)

[3] Ishihara, K., Nakaoka, M. and Nishioka, M., Study on a countermeasure for high level sound generated from boiler tube bank duct using walls made of perforated plate (In case of aperture ratio being more than 1\%), Transactions of the J SME (in Japanese), Vol.82, No.841 (2016), DOI: 10.1299/transjsme. 16-00179.

[4] Ishihara, K., Study on a countermeasure for high level sound generated from boiler tube bank duct using walls made of perforated plate (Grasp critical aperture ratio and influence of cavity volume on suppression effect), Transactions of the JSME (in Japanese), Vol.83, No.848 (2017), DOI: 10.1299/transjsme. 16-00456

[5] Ishihara, K., Kudo, S., Masumoto, T. and Mori, M., Acoustic natural frequency of one dimensional sound field partitioned by perforated plate (comparison between analysis and experiment), Proceedings of the 55th Annular Meeting in Chugoku-Shikoku (2017), ID: K1114 (in Japanese).

[6] Ishihara, K., Kudo, S., Masumoto, T. and Mori, M., Study on acoustic natural frequency and its mode of one dimensional sound field partitioned by perforated plate, Transactions of the JSME (in Japanese), Vol.84, No.857 (2018), DOI:10. 1299/transjsme. 17-00365.

[7] Kudo, S. and Ishihara, K., Effect of perforated plate on acoustic natural frequency of one dimensional sound field partitioned by perforated plate, 27th Environment engineering symposium paper (2017), ID: 111(in Japanese)

[8] Kudo, S. and Ishihara, K., Effect of set position of perforated plate on acoustic natural frequency of one dimensional sound field, 28th Environment engineering symposium paper (2018), ID: 128(in Japanese).

[9] Melling, T. H., The acoustic impedance of perforates at medium and high sound pressure levels, Journal of Sound and Vibration, Vol.29, No.1 (1973), pp.1-65.

[10] Utsuno, H., Itaya, T., Experimental study of Sound Absorbing characteristics for perforated panel by Four-microphone measuring technique, Proceedings of JSME Dynamics and Design Conference 2001, on CD-ROM (in Japanese).

Kunihiko Ishihara was born in 1947 in Kurashiki City, Okayama Prefecture Japan. He received the B.S. degree from Kobe University in 1969. He got a master's degree in Kobe University in 1971 and earned the Ph.D. degree in Engineering from The Osaka University in 1986.

$\mathrm{He}$ worked in Kawasaki Heavy Industry Co. Ltd. as an Mechanical Engineer for 33 years. After that he became a Professor of The University of Tokushima in 2004. He had been studying the vibration and noise control, above all he studied the flow induced vibration and noise problems. He has authored or co-authored over 100 technical journal and over 50 conference papers. He is a fellow of JSME (Japan Society of Mechanical Engineers) now. He is a Professor of Tokushima Bunri University. He teaches a mechanical field subjects for students.

Akari Goto was born in 1984 in Tonosho, Kagawa Prefecture Japan. She received a bachelor's degree (clinical engineering) from Hiroshima International University in 2007. She got a master's degree in 2009 and earned the Ph.D. degree in Medical Engineering from The Hiroshima International University in 2012.

She became a lecturer at Tokushima Bunri University in 2009. And she has been a teacher since 2011 .

She conducts research on blood coagulation, medical materials, and medical device development.

Makoto Kashino was born in 1976 in Fukuyama City, Hiroshima Prefecture Japan. He received a bachelor's degree (clinical engineering) from Hiroshima International University in 2002. He got a master's degree in 2005 and earned the Ph.D. degree in Medical Engineering from The Hiroshima International University in 2018.

He worked for 6 years as a clinical engineer at Teraoka Memorial Hospital and Nippon Kokan Fukuyama Hospital. After that he became a lecturer at Tokushima Bunri University in 2008. And he has been an associate professor since 2018.

He conducts research on Hemodialysis therapy, Medical gas and Medical device development. 